\title{
Contribution of the thermotolerance genomic island to increased thermal tolerance in Cronobacter strains
}

\author{
Maria Orieskova1 $^{1}$, Michal Kajsik ${ }^{1}$, Tomas Szemes ${ }^{1}$, Ondrej Holy ${ }^{2}$, Stephen Forsythe ${ }^{3}$, \\ Jan Turna ${ }^{1}$, Hana Drahovska ${ }^{1}$
}

${ }^{1}$ Department of Molecular Biology, Faculty of Natural Sciences, Comenius University, Bratislava, Slovak Republic

${ }^{2}$ Department of Preventive Medicine, Faculty of Medicine and Dentistry, Palacký University, Olomouc, Olomouc, Czech Republic

${ }^{3}$ Pathogen Research Group, School of Science and Technology, Nottingham Trent University, Clifton Lane, Nottingham NG11 8NS, UK

Correspondence to: Hana Drahovská, Department of Molecular Biology, Faculty of Natural Sciences, Comenius University, Ilkovičova 6, 84115 Bratislava, Slovakia;

e-mail: drahovska@fns.uniba.sk

Telephone number: 42126096639

Fax number: 42126096508

The manuscript accepted in Antonie van Leeuwenhoek, International Journal of General and Molecular Microbiology 


\begin{abstract}
Cronobacter spp. are opportunistic pathogens associated with serious infections in neonates. Increased stress tolerance, including the thermotolerance of some Cronobacter strains, can promote their survival in production facilities and thus raise the possibility of contamination of dried infant formula which has been identified as a potential source of infection. Some Cronobacter strains contain a genomic island, which might be responsible for increased thermotolerance. By analysis of Cronobacter sequenced genomes this determinant was found to be present in only 49/73 Cronobacter sakazakii strains and in 9/14 Cronobacter malonaticus strains. The island was also found in 16/17 clinical isolates originating from two hospitals. Two configurations of the locus were detected; the first one with the size of $18 \mathrm{kbp}$ containing the thrB-Q genes and a shorter version $(6 \mathrm{kbp})$ harbouring only the thr BCD and thr $\mathrm{OP}$ genes. Strains containing the thermotolerance island survived significantly better at $58^{\circ} \mathrm{C}$ comparing to a $C$. sakazakii isogenic mutant lacking the island and strains with the longer version of the island were 2-10 times more tolerant than those with the shortened sequence. The function of the genomic island was further confirmed by its cloning into a low-copy vector and transforming it into the isogenic mutant. Different levels of rpoS, encoding for stress-response sigma factor, expression were also associated with variability in strain thermotolerance.
\end{abstract}

Keywords: Cronobacter spp., heat stress, rpoS, thermotolerance, thermotolerance island 


\section{Introduction}

Cronobacter spp. are Gram-negative, rod shaped bacteria from the family Enterobacteriaceae. They were formerly known as Enterobacter sakazakii and were classified as a separate genus in 2007 (Iversen et al. 2007). Recently the genus Cronobacter has undergone a number of revisions and currently contains seven species (Iversen et al. 2008; Joseph et al. 2012; Forsythe et al. 2014; Stephan et al. 2014). Cronobacter strains are opportunistic pathogens that can cause serious infections in neonates, including meningitis, necrotising enterocolitis and sepsis with low frequency, but high lethality rate (Hunter and Bean 2013; Holy and Forsythe 2014). Infections in adults also have been reported, in particular among the elderly and immunocompromised patients (Patrick et al. 2014; Alsonosi et al. 2015).

Whereas Cronobacter strains are ubiquitous and have been isolated from various foods and environments (Turcovsky et al. 2011; Holy and Forsythe 2014) the main vehicle for their transmission in neonatal infections is rehydrated powdered infant formula (PIF) (Yan et al. 2012; Holy and Forsythe 2014). In comparison with other Enterobacteriaceae, members of the genus Cronobacter are considerably resistant to desiccation (Caubilla-Barron and Forsythe 2007; Osaili and Forsythe 2009; Walsh et al. 2011; Jaradat et al. 2014) and this property contributes to their survival in PIF. Thermal tolerance is another important factor for survival of Cronobacter in food (Dancer et al. 2009; Jaradat et al. 2014; Huertas et al. 2015). It has been shown that Cronobacter strains differ in their tolerance to heat stress. Edelson-Mammel and Buchanan (2004) obtained decimal reduction times at $58^{\circ} \mathrm{C}\left(\mathrm{D}_{58}\right)$ in the range from 0.5 to 9.9 min and the Cronobacter strains fell into two heat resistance phenotypes, thermosensitive and thermotolerant. A protein marker unique for thermotolerant Cronobacter strains was later identified by mass spectrometry (Williams et al. 2005). In our previous work, we found that this thermotolerance marker is encoded by a gene localised on a genomic island which contained a cluster of conserved genes encoding several stress response proteins (Gajdosova et al. 2011). We observed that an isogenic mutant lacking the thermotolerance island was defective in survival at increased temperatures both in exponential and stationary growth phase (Orieskova et al. 2013).

The aim of the present study was to compare the composition of the thermotolerance genomic island in genome sequenced Cronobacter strains and to correlate the genomic differences with their phenotypes.

\section{Materials and Methods}

Bacterial strains used in the study. Cronobacter collection strains were obtained from the Belgian Co-ordinated Collections of Microorganisms (BCCM/LMG) and from Nottingham Trent University UK. C. sakazakii JG-05 was prepared from C. sakazakii ATCC $29544^{\mathrm{T}}$ by deletion of the whole genomic island (Orieskova et al. 2013). Clinical Cronobacter strains were isolated during 2012-2013 from two hospitals in the Czech Republic (Alsonosi et al. 2015). Strains and plasmids used in the study are listed in Tables S1 and S2. All strains were maintained in LB medium supplemented with $15 \%$ glycerol at $-80^{\circ} \mathrm{C}$ and routinely cultured on $\mathrm{LB}$ medium $/ \mathrm{LB}$ agar at $37^{\circ} \mathrm{C}$.

Detection of genes from the thermotolerance island in Cronobacter genomes. Cronobacter genomes deposited in the PubMLST database (http://pubmlst.org/cronobacter/) were used as the source of genomic sequences for analyses (Jolley and Maiden 2010; Forsythe et al. 2014). Genes thrB-Q from the C. sakazakii ATCC 29544 ${ }^{\mathrm{T}}$ thermotolerance island (FR714908) and thrOP from $C$. sakazakii NTU701 were Blast searched against the database and hits with sufficient coverage and similarity (both up to $90 \%$ on nucleotide level) were scored as positive. 
Indirect assays for the assessment of RpoS status. The catalase activity of $C$. sakazakii strains was measured according to Alvarez-Ordonez et al. (2012) by dropping $20 \mu \mathrm{l}$ of $6 \%$ hydrogen peroxide onto fresh colonies that were grown on LB agar for $24 \mathrm{~h}$ at $37^{\circ} \mathrm{C}$. Intensity of bubbling indicated level of catalase activity. Yellow pigmentation of colonies was assessed using cultures grown on LB-PIF agar, which was prepared by mixing of one volume of 2-times concentrated LB-agar and one volume of reconstituted PIF. LB-PIF plates were grown at $37^{\circ} \mathrm{C}$ for $24 \mathrm{~h}$ in a dark incubator followed by $48 \mathrm{~h}$ further incubation in daylight at room temperature.

Determination of thermotolerance. $100 \mu \mathrm{l}$ overnight bacterial culture (approximately $18 \mathrm{~h}$ at $37^{\circ} \mathrm{C}$ ) was mixed with $900 \mu \mathrm{l}$ fresh pre-heat LB medium and incubated at $58^{\circ} \mathrm{C}$. Aliquots were withdrawn into sterile $200 \mu \mathrm{l}$ thin-wall microtubes and chilled on ice for $1 \mathrm{~min}$ and the numbers of surviving cells were determined by the plate-count procedure. Each value was determined in triplicate for each culture.

Cloning of the thermotolerance island. The island was amplified from genomic DNA using CloneAmp Hifi PCR Premix (Clontech) and primers pThr2118F-HindIII (ataagcttacggatcgtgcccattattacg) and pThr17274R-HindIII (acgaagcttgccetgtcgaacctatggat) for C. sakazakii ATCC $29544^{\mathrm{T}}$ strain and pThr2118F-HindIII and pCs701-R1-HindIII (ccaagcttctgtaggggtcgggtaggtc) for C. sakazakii NTU696 and C. sakazakii NTU701 strains. Vector DNA (origin, Cm cassette) was amplified from the pACYCDuet1 (Novagen) with primers VecD2f-Thr2f (cgatccgtaagcttatagaagcacacggtcacactg) and VecD2r-Thr2r (acagggcaagcttcgtttcaacccagtcagctcctt) or with VecD2f-Thr2f and VecD2r-Thr701R (ccctacagaagcttggttcaacccagtcagctcctt) respectively. The In-Fusion ${ }^{\circledR}$ HD EcoDry ${ }^{\mathrm{TM}}$ Cloning Kit (Clontech) was used for direct joining of the resulting fragments in recombinant plasmids pACYCThr2-ATCC29544, pACYCThr2-NTU696 and pACYCThr2-NTU701 (Fig.1, Table S2). The PCR product obtained by pACYCDuet1 amplification with primers VecD2f-Thr2f /VecD2r-Thr2r digested by HindIII was ligated to prepare control plasmid pACYCK2.

Cloning of rpoS gene. The gene was amplified from Escherichia coli KL53, an uropathogenic strain, with primers RpoSF (agcgctagcatgagtcagaatacgctgaaagttc) and RpoSR (gatgaattcttactcgcggaacagcg). The PCR product was digested with EcoRI and NheI and cloned into vector prepared by digestion of the pBAD-GFPuv plasmid with the same enzymes (Table S2). The same primers were used for rpoS sequencing in $C$. sakazakii NTU696. 


\section{Results and Discussion}

\section{Comparison of thermotolerance islands in Cronobacter strains}

The aim of this study was to characterise the thermal tolerance which is mediated by the genomic island present in some Cronobacter strains. The island from the C. sakazakii ATCC $29544^{\mathrm{T}}$ type strain, which is $18 \mathrm{kbp}$ long and harbours thrB-Q genes (Fig. 1, Table S3), was described in our previous studies (Gajdosova et al. 2011; Orieskova et al. 2013). Recently, genomes of fourteen Cronobacter strains covering seven species were published (Joseph et al. 2012) and a comparison with C. sakazakii ATCC $29544^{\mathrm{T}}$ showed that the genes from the thermotolerance island were found in two from five $C$. sakazakii (NTU696 and NTU701) and in both C. malonaticus (LMG23826 ${ }^{\mathrm{T}}$ and NTU507) genomes from this study. C. malonaticus strains contained thermotolerance islands of similar size and organisation as that present in $C$. sakazakii ATCC $29544^{\mathrm{T}}$. However, the genomes of $C$. sakazakii NTU696 and $C$. sakazakii NTU701 possessed a version of the island shortened to $6 \mathrm{kbp}$, having only thrBCD and thrOP genes from, respectively, the beginning and the end of the original version (Fig 1). The similarity of the thrBCD gene products from the long and shortened islands was high $(93 \%$ $99 \%$ at the amino acid level), but the thrOP gene products showed only moderate similarity ( $76 \%$ and $77 \%$ at the amino acid level for ThrO and ThrP respectively).

The number of sequenced genomes is steadily increasing and 108 Cronobacter genome sequences representing all seven species of the genus have been deposited in the Cronobacter PubMLST database (http://pubmlst.org/cronobacter/) at the time of analysis (May 2015) (Forsythe et al. 2014). In agreement with our previous observations, the thermotolerance island was present only in C. sakazakii (in 49 from 73 strains) and in C. malonaticus (in 9 from 14 strains) deposited in the database (Table S4). Twenty-three of them (14 C. sakazakii and all 9 C. malonaticus) contained the full length version of the island, while thirty-five C. sakazakii strains possessed the shortened one. We observed high sequence conservation in the locus; the similarity at the DNA level reached $99.5-100 \%$ between strains with the same type of the island. By cluster analysis sequences of the long islands were separated into two groups: the first contained the majority of the $C$. malonaticus strains; $C$. sakazakii and the one remaining C. malonaticus strain belonged to the second cluster (Fig. S1). Strains containing the short island were separated into several smaller groups in agreement with their origin and MLST sequence type (Fig. S2).

A genomic region with a high degree of sequence similarity was recently described also in thermotolerant Klebsiella pneumoniae strains responsible for nosocomial infections due to increased resistance to disinfection protocols (Bojer et al. 2010) and in several heat resistant E. coli strains isolated from beef samples (Mercer et al. 2015).

Thermotolerant Cronobacter strains originated from various sources, mainly from clinical samples (73\%), PIF and formula production facilities (14\%) and from the environment (13\%). By comparing the genomic island occurrence in the MLST sequence types we observed that the majority of the strains belonged to the ST4 (61\%), which is a virulent clonal lineage responsible for most of the meningitis cases in newborns (Joseph and Forsythe 2011; Forsythe et al. 2014). Several other positive $C$. sakazakii strains were classified as ST8, ST12 and ST13, which are also associated with infections in neonates and adults. For example, the $C$. sakazakii ATCC $29544^{\mathrm{T}}$ type strain, which belongs to ST8, was isolated from human throat and the ST12 and ST13 strains were isolated during an NICU outbreak in France in 1994 (Caubilla-Barron et al. 2007); during this epidemic ST12 strains were associated with necrotizing enterocolitis cases (Forsythe et al. 2014; Masood et al. 2015). The nine C. malonaticus strains containing the thermotolerance island belonged to five different sequence types. However, four ST7 strains were the most prevalent found among these strains and this sequence type is connected with 
adult infections, especially in immunocompromised and elderly patients (Forsythe et al. 2014; Alsonosi et al. 2015).

\section{Thermotolerance in selected strains}

Subsequently, the thermal tolerance of $C$. sakazakii and $C$. malonaticus strains obtained from the study described by Joseph et al. (2012) were compared with that of the $C$. sakazakii type strain ATCC $29544^{\mathrm{T}}$. The mutant $C$. sakazakii JG-05, prepared from $C$. sakazakii ATCC $29544^{\mathrm{T}}$ by deletion of the whole thermotolerance island and described in our previous study (Orieskova et al. 2013) was used as a control (Fig. 2). We observed that strains containing the thermotolerance island survived significantly better at $58^{\circ} \mathrm{C}$ compared with the deletion mutant and that $C$. sakazakii ATCC $29544^{\mathrm{T}}$ possessed the highest thermotolerance of all the strains, which is in agreement with our previously reported results (Gajdosova et al. 2011). Strains with the longer version of the island (C. sakazakii ATCC $29544^{\mathrm{T}}$, C. malonaticus LMG $23826^{\mathrm{T}}$ and NTU507) were 2-10 times more thermotolerant than strain $C$. sakazakii NTU701 containing the sequence shortened to the thrBCD and thrOP genes. This observation confirmed the results of Bojer et al. (2013), who studied K. pneumoniae strains containing a similar thermotolerance region and showed that thermotolerance could differ according to the presence of the longer or shorter version of the locus. The $C$. sakazakii NTU696 strain was the only exception, as it contained a thermotolerance island with an almost identical sequence to that of $C$. sakazakii NTU701, but its survival ability was lower than that of the deletion mutant $C$. sakazakii JG-05 (Fig. 2). We concluded that the lowered heat stability of $C$. sakazakii NTU696 must be caused by some genetic defect occurred outside from the thermotolerance island (as shown below).

\section{Cloning of the thermotolerance island}

The function of the thermotolerance island was further confirmed by cloning it into a low copy plasmid vector. Three constructs, pACYCThr2-ATCC29544, pACYCThr2-NTU696 and pACYCThr2-NTU701, were prepared and transformed into the $C$. sakazakii JG-05 deletion mutant (Fig. 1, Table S2). We observed significantly higher survival of all three constructs at $58^{\circ} \mathrm{C}$ compared with a control containing the vector only (Fig. 3a), which further proved the role of this region in increased thermal tolerance. The pACYCThr2-ATCC29544 plasmid enhanced the thermal tolerance of $C$. sakazakii JG-05 seventeen times. However, by comparing with the parent strain $C$. sakazakii ATCC $29544^{\mathrm{T}}$, the complemented mutant $C$. sakazakii JG05 pACYCThr2-ATCC29544 showed four times reduced survival. We propose that lowered thermotolerance of the complemented mutant was caused either by polar effects or by impaired regulation of gene expression as excessive production of heat shock proteins could be toxic for the cell (Battesti et al. 2011). Similar to the results with the original strains, the $C$. sakazakii JG-05 complemented with the shortened version of the island (pACYCThr2-NTU696 or pACYCThr2-NTU701) showed increased survival comparing to the non-complemented strain but it was not as thermotolerant as the strain transformed with the full version of the island (Fig. 3a). We observed that, unlike the parent strain C. sakazakii NTU696, the pACYCThr2NTU696 plasmid was able to increase thermotolerance of the complemented strain to a similar extent as the construct pACYCThr2-NTU701. These data strongly suggest that the difference in thermal tolerance between C. sakazakii NTU696 and NTU701 was caused by genetic determinants outside of the island. The constructed plasmids were also transformed into E. coli DH5a (Fig 3b). We observed better survival of the E. coli control strain compared to $C$. sakazakii JG-05 but the presence in this strain of the thermotolerance constructs also significantly (seven times) increased heat tolerance. It is also noteworthy that no difference between the plasmids containing short and long versions of the island was seen in E. coli (Fig 3b). By analysis of thermotolerance in Klebsiella strains (Bojer et al. 2013) found out that resistance mediated by the $c l p \mathrm{~K}(t h r \mathrm{D})$ gene alone is dependent on the presence of a $c l p \mathrm{P}$ 
background, but $c l p \mathrm{P}$ was not necessary for thermotolerance mediated by the entire heat tolerance locus encompassing $c l p K$. In our screening, 104/108 Cronobacter genomes deposited in the MLST database (May 2015) possessed the cplP gene. In the study by Huertas et al. (2015), a Cronobacter clpP mutant was found to be attenuated in thermotolerance by screening of a transposon library originating from an environmentally resistant strain. However, it is not known if the thermotolerance island is present in this strain. The presence of the long version of the thermotolerance island correlated with heat resistance in E. coli and complementation generating thermotolerance was achieved only if the entire genomic island was cloned into a sensitive E. coli strain and thus the thermoresistance was dependent on the entire genomic island, and not on the function of a single protein (Mercer et al. 2015).

\section{Complementation of rpoS gene}

According to the study by Alvarez-Ordonez et al. (2012), Cronobacter strains expressed variable levels of $r p o S$ gene encoding for stress-response sigma factor $\left(\sigma^{38}\right)$ and this variability was responsible for differences in many phenotypic properties between strains. As the resistance to heat stress is also influenced by the level of RpoS we checked the status of this gene in our strains. We measured production of catalase and yellow pigmentation as markers of RpoS activity (Alvarez-Ordonez et al. 2012) and found out that the RpoS level in C. sakazakii NTU701 was apparently high because of high production of oxygen from peroxide and yellow pigmentation of colonies. However, zero activity was detected in C. sakazakii NTU696 by these two tests suggesting non-functional rpoS. We detected that a premature stop codon in the rpoS gene (G443A mutation) was responsible for this property. By transformation of an E. coli rpoS mutant with the thermotolerance island plasmids we observed increased thermotolerance in transformed strains but the cell survival was lower than that of the same constructs in wild-type E. coli (data not shown). This experiment confirmed our previous observations that genes from the thermotolerance island are expressed in both exponential and stationary phases but exponentially grown bacteria are not as tolerant as stationary bacteria (Gajdosova et al. 2011; Orieskova et al. 2013). Subsequently, a recombinant plasmid containing rpoS from E. coli under the control of the arabinose promoter was constructed and used to complement the $C$. sakazakii NTU696 strain. We observed that the heat survival of $C$. sakazakii NTU696 pBADrpoS reached the level of the $C$. sakazakii NTU701 containing the same thermotolerance island and the functional rpoS gene (Fig. 4) and thus we confirmed the role of rpoS mutation in the lowered survival of $C$. sakazakii NTU696 at high temperatures.

\section{Thermotolerance of clinical Cronobacter strains}

In the last part of the present study we characterised the thermotolerance of clinical Cronobacter strains. Seventeen strains isolated during 2012-2013 in two hospitals in the Czech Republic and described previously (Alsonosi et al. 2015) were used in the experiments. The presence of three genes ( $t h r \mathrm{D}, t h r \mathrm{I}, t h r \mathrm{~J})$ from the genomic island was tested in these strains by PCR. A positive signal for all three markers was obtained for ten $C$. sakazakii ST4 and six $C$. malonaticus ST7 strains. The single $C$. sakazakii ST64 strain tested was negative for all three markers. In next step, strain survival was tested at $58^{\circ} \mathrm{C}$ (Fig. 5). In agreement with our previous experiments, we observed that strains with the thermotolerance island survived 2-11 times better compared with the $C$. sakazakii JG-05 deletion mutant. At the same time, thermotolerant C. sakazakii strains survived better than clinical isolates belonging to C. malonaticus and thus confirmed our data obtained with culture collection strains.

The frequency of the thermotolerance island in hospital isolates was quite high compared with its occurrence in strains from other environments. For example only up to $10 \%$ (6/76) of C. sakazakii and C. malonaticus isolated from food in our previous studies were positive for the thrI marker (Turcovský et al. 2011, Gajdošová et al. 2011, Gičová et al. 2013). 
The island is also frequently present in genomes of ST4 and ST7 strains which are Cronobacter clones possessing increased virulence (Joseph and Forsythe 2011; Forsythe et al. 2014). Previously, K. pneumoniae strains containing genes homologous to the genomic island were described to cause nosocomial outbreaks as they were able to survive routine disinfection processes (Bojer et al. 2010). We suppose that similar mechanisms could be responsible for spreading of the thermotolerant Cronobacter strains in hospitals. Another interesting observation was that Cronobacter strains lacking the thermotolerance genomic island are significantly less tolerant to heat stress compared with $E$. coli (Fig. 3a and 3b) and therefore we propose that a relatively high frequency of this genetic element in C. sakazakii and C. malonaticus presents a compensation mechanism for this defect.

\section{Conclusion.}

In this study, the contribution of the thermotolerance genomic island to the increased thermal tolerance in some Cronobacter strains was confirmed. We observed that the full-length island containing the thrB-Q genes was more efficient than its shortened version. Different levels of rpoS expression were found to contribute to variability in thermotolerance. The presence of the thermotolerance island likely improves the ability of positive Cronobacter strains to survive in the PIF, food production facility and hospital environments.

\section{Acknowledgement}

This publication is the result of the project implementation (ITMS 26240220086) supported by the Research \& Development Operational Programme funded by the ERDF and by Slovak Ministry of Education under the contract No. VEGA 1/0709/12. Project supported by Research Support Foundation, Vaduz - grant project nr. 801100021/39 - Surveillance of Infectious Complications in Hemato-Oncological Patients.

\section{References}

Alsonosi A, Hariri S, Kajsik M, Orieskova M, Hanulik V, Roderova M, Petrzelova J, Kollarova H, Drahovska H, Forsythe S, Holy O (2015) The speciation and genotyping of Cronobacter isolates from hospitalised patients. Eur J Clin Microbiol Infect Dis 34:1979-1988

Alvarez-Ordonez A, Begley M, Hill C (2012) Polymorphisms in rpoS and stress tolerance heterogeneity in natural isolates of Cronobacter sakazakii. Appl Environ Microbiol 78:3975-3984

Battesti A, Majdalani N, Gottesman S (2011) The RpoS-mediated general stress response in escherichia coli. Annu Rev Microbiol 65:189-213

Bojer MS, Struve C, Ingmer H, Hansen DS, Krogfelt KA (2010) Heat Resistance Mediated by a New Plasmid Encoded Clp ATPase, ClpK, as a Possible Novel Mechanism for Nosocomial Persistence of Klebsiella pneumoniae. PLoS ONE 5:e15467

Bojer MS, Struve C, Ingmer H, Krogfelt KA (2013) ClpP-dependent and -independent activities encoded by the polycistronic clpK-encoding locus contribute to heat shock survival in Klebsiella pneumoniae. Res Microbiol 164:205-210

Caubilla-Barron J, Forsythe S (2007) Dry Stress and Survival Time of Enterobacter sakazakii and Other Enterobacteriaceae in Dehydrated Powdered Infant Formula. J Food Prot 70:2111-2117

Caubilla-Barron J, Hurrell E, Townsend S, Cheetham P, Loc-Carrillo C, Fayet O, Prere MF, Forsythe SJ (2007) Genotypic and phenotypic analysis of Enterobacter sakazakii 
strains from an outbreak resulting in fatalities in a neonatal intensive care unit in France. J Clin Microbiol 45:3979-3985

Dancer GI, Mah JH, Rhee MS, Hwang IG, Kang DH (2009) Resistance of Enterobacter sakazakii (Cronobacter spp.) to environmental stresses. J Appl Microbiol 107:16061614

Edelson-Mammel SG, Buchanan RL (2004) Thermal Inactivation of Enterobacter sakazakii in Rehydrated Infant Formula. J Food Prot 67:60-63

Forsythe S, Dickins B, Jolley KA (2014) Cronobacter, the emergent bacterial pathogen Enterobacter sakazakii comes of age; MLST and whole genome sequence analysis. BMC Genomics 15:1121

Gajdosova J, Benedikovicova K, Kamodyova N, Tothova L, Kaclikova E, Stuchlik S, Turna J, Drahovska H (2011) Analysis of the DNA region mediating increased thermotolerance at $58^{\circ} \mathrm{C}$ in Cronobacter sp. and other enterobacterial strains. Antonie Van Leeuwenhoek 100:279-289

Holy O, Forsythe S (2014) Cronobacter spp. as emerging causes of healthcare-associated infection. J Hosp Infect 86:169-177

Huertas J-P, Álvarez-Ordóñez A, Morrissey R, Ros-Chumillas M, Esteban M-D, Maté J, Palop A, Hill C (2015) Heat resistance of Cronobacter sakazakii DPC 6529 and its behavior in reconstituted powdered infant formula. Food Research International 69:401-409

Hunter CJ, Bean JF (2013) Cronobacter: an emerging opportunistic pathogen associated with neonatal meningitis, sepsis and necrotizing enterocolitis. J Perinatol 33:581-585

Iversen C, Lehner A, Mullane N, Bidlas E, Cleenwerck I, Marugg J, Fanning S, Stephan R, Joosten H (2007) The taxonomy of Enterobacter sakazakii: proposal of a new genus Cronobacter gen. nov. and descriptions of Cronobacter sakazakii comb. nov.

Cronobacter sakazakii subsp. sakazakii, comb. nov., Cronobacter sakazakii subsp. malonaticus subsp. nov., Cronobacter turicensis sp. nov., Cronobacter muytjensii sp. nov., Cronobacter dublinensis sp. nov. and Cronobacter genomospecies 1. BMC Evol Biol 7:64

Iversen C, Mullane N, McCardell B, Tall BD, Lehner A, Fanning S, Stephan R, Joosten H (2008) Cronobacter gen. nov., a new genus to accommodate the biogroups of Enterobacter sakazakii, and proposal of Cronobacter sakazakii gen. nov., comb. nov., Cronobacter malonaticus sp. nov., Cronobacter turicensis sp. nov., Cronobacter muytjensii sp. nov., Cronobacter dublinensis sp. nov., Cronobacter genomospecies 1, and of three subspecies, Cronobacter dublinensis subsp. dublinensis subsp. nov., Cronobacter dublinensis subsp. lausannensis subsp. nov. and Cronobacter dublinensis subsp. lactaridi subsp. nov. Int J Syst Evol Microbiol 58:1442-1447

Jaradat ZW, Al Mousa W, Elbetieha A, Al Nabulsi A, Tall BD (2014) Cronobacter spp.-opportunistic food-borne pathogens. A review of their virulence and environmentaladaptive traits. J Med Microbiol 63:1023-1037

Jolley KA, Maiden MC (2010) BIGSdb: Scalable analysis of bacterial genome variation at the population level. BMC Bioinformatics 11:595

Joseph S, Cetinkaya E, Drahovska H, Levican A, Figueras MJ, Forsythe SJ (2012) Cronobacter condimenti sp. nov., isolated from spiced meat, and Cronobacter universalis sp. nov., a species designation for Cronobacter sp. genomospecies 1, recovered from a leg infection, water and food ingredients. Int J Syst Evol Microbiol 62:1277-1283

Joseph S, Desai P, Ji Y, Cummings CA, Shih R, Degoricija L, Rico A, Brzoska P, Hamby SE, Masood N, Hariri S, Sonbol H, Chuzhanova N, McClelland M, Furtado MR, Forsythe 
SJ (2012) Comparative analysis of genome sequences covering the seven Cronobacter species. PLoS One 7:e49455

Joseph S, Forsythe SJ (2011) Predominance of Cronobacter sakazakii sequence type 4 in neonatal infections. Emerg Infect Dis 17:1713-1715

Masood N, Moore K, Farbos A, Paszkiewicz K, Dickins B, McNally A, Forsythe S (2015) Genomic dissection of the 1994 Cronobacter sakazakii outbreak in a French neonatal intensive care unit. BMC Genomics 16:750

Mercer RG, Zheng J, Garcia-Hernandez R, Ruan L, Ganzle MG, McMullen LM (2015) Genetic determinants of heat resistance in Escherichia coli. Front Microbiol 6:932

Orieskova M, Gajdosova J, Oslanecova L, Ondreickova K, Kaclikova E, Stuchlik S, Turna J, Drahovska H (2013) Function of thermotolerance genomic island in increased stress resistance of Cronobacter sakazakii. Journal of Food and Nutrition Research 52:37-44

Osaili T, Forsythe S (2009) Desiccation resistance and persistence of Cronobacter species in infant formula. Int J Food Microbiol 136:214-220

Patrick ME, Mahon BE, Greene SA, Rounds J, Cronquist A, Wymore K, Boothe E, Lathrop S, Palmer A, Bowen A (2014) Incidence of Cronobacter spp. infections, United States, 2003-2009. Emerg Infect Dis 20:1520-1523

Stephan R, Grim CJ, Gopinath GR, Mammel MK, Sathyamoorthy V, Trach LH, Chase HR, Fanning S, Tall BD (2014) Re-examination of the taxonomic status of Enterobacter helveticus, Enterobacter pulveris and Enterobacter turicensis as members of the genus Cronobacter and their reclassification in the genera Franconibacter gen. nov. and Siccibacter gen. nov. as Franconibacter helveticus comb. nov., Franconibacter pulveris comb. nov. and Siccibacter turicensis comb. nov., respectively. Int J Syst Evol Microbiol 64:3402-3410

Turcovsky I, Kunikova K, Drahovska H, Kaclikova E (2011) Biochemical and molecular characterization of Cronobacter spp. (formerly Enterobacter sakazakii) isolated from foods. Antonie Van Leeuwenhoek 99:257-269

Walsh D, Molloy C, Iversen C, Carroll J, Cagney C, Fanning S, Duffy G (2011) Survival characteristics of environmental and clinically derived strains of Cronobacter sakazakii in infant milk formula (IMF) and ingredients. J Appl Microbiol 110:697-703

Williams TL, Monday SR, Edelson-Mammel S, Buchanan R, Musser SM (2005) A top-down proteomics approach for differentiating thermal resistant strains of Enterobacter sakazakii. Proteomics 5:4161-4169

Yan QQ, Condell O, Power K, Butler F, Tall BD, Fanning S (2012) Cronobacter species (formerly known as Enterobacter sakazakii) in powdered infant formula: a review of our current understanding of the biology of this bacterium. J Appl Microbiol 113:1-15 
Fig 1. Plasmid constructs containing the thermotolerance islands from Cronobacter strains

Genes B-Q originated from the thermotolerance islands of the strains C. sakazakii ATCC $29544^{\mathrm{T}}$, C. sakazakii NTU696 and C. sakazakii NTU701. p15A origin and chloramphenicol resistance gene $(\mathrm{CmR})$ were from pACYC-Duet. Numbers in bottom line represent protein similarity between $C$. sakazakii NTU696 and C. sakazakii ATCC $29544^{\mathrm{T}}$. Similarity of proteins from $C$. sakazakii NTU701 and C. sakazakii NTU696 is $99.5-100 \%$. Predicted gene functions are listed in Table S3.

pACYCThr2-ATCC29544

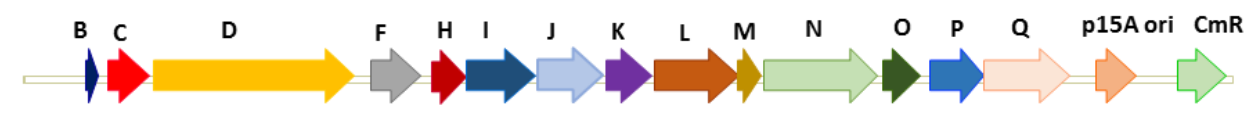

pACYCThr2-NTU701
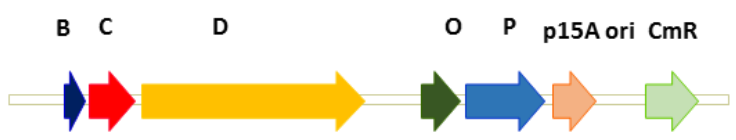

pACYCThr2-NTU696

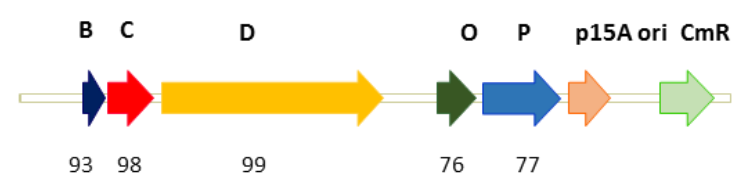


Fig 2. Thermotolerance of Cronobacter strains

Labelling of the strains: C. sakazakii ATCC $29544^{\mathrm{T}}$ (filled circles), C. sakazakii NTU701 (filled triangles), C. sakazakii NTU 696 (empty triangles), C. sakazakii JG-05 (crosses), C. malonaticus LMG $23826^{\mathrm{T}}$ (empty diamonds), C. malonaticus NTU507 (filled diamonds). Survival of stationary phase cells was determined at $58^{\circ} \mathrm{C}$.

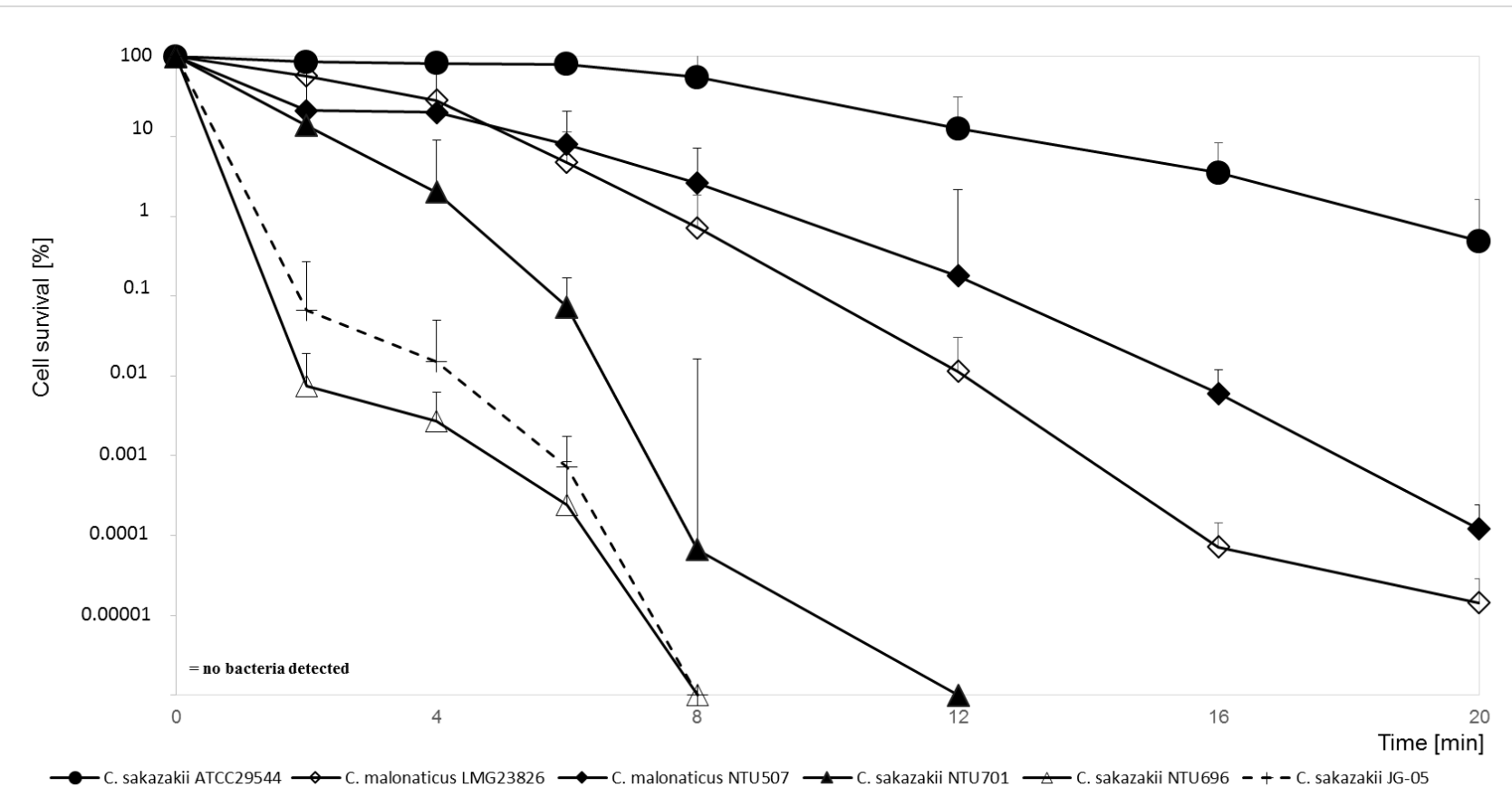


Fig 3. Survival of $E$. coli and $C$. sakazakii strains complemented with plasmids encoding for the thermotolerance islands

A) C. sakazakii JG-05 (a deletion mutant lacking the thermotolerance island) was transformed with plasmids pACYC-ATCC29544 (filled circles), pACYC-NTU701 (filled triangles) and pACYC-NTU696 (empty triangles) encoding for the thermotolerance islands from the respective Cronobacter strains (Fig. 1). C. sakazakii ATCC $29544^{\mathrm{T}}$ (empty circles) and C. sakazakii JG-05 transformed with empty vector pACYCK2 (crosses) were used as positive and negative controls, respectively. Survival of stationary phase cells was determined at $58^{\circ} \mathrm{C}$.

A

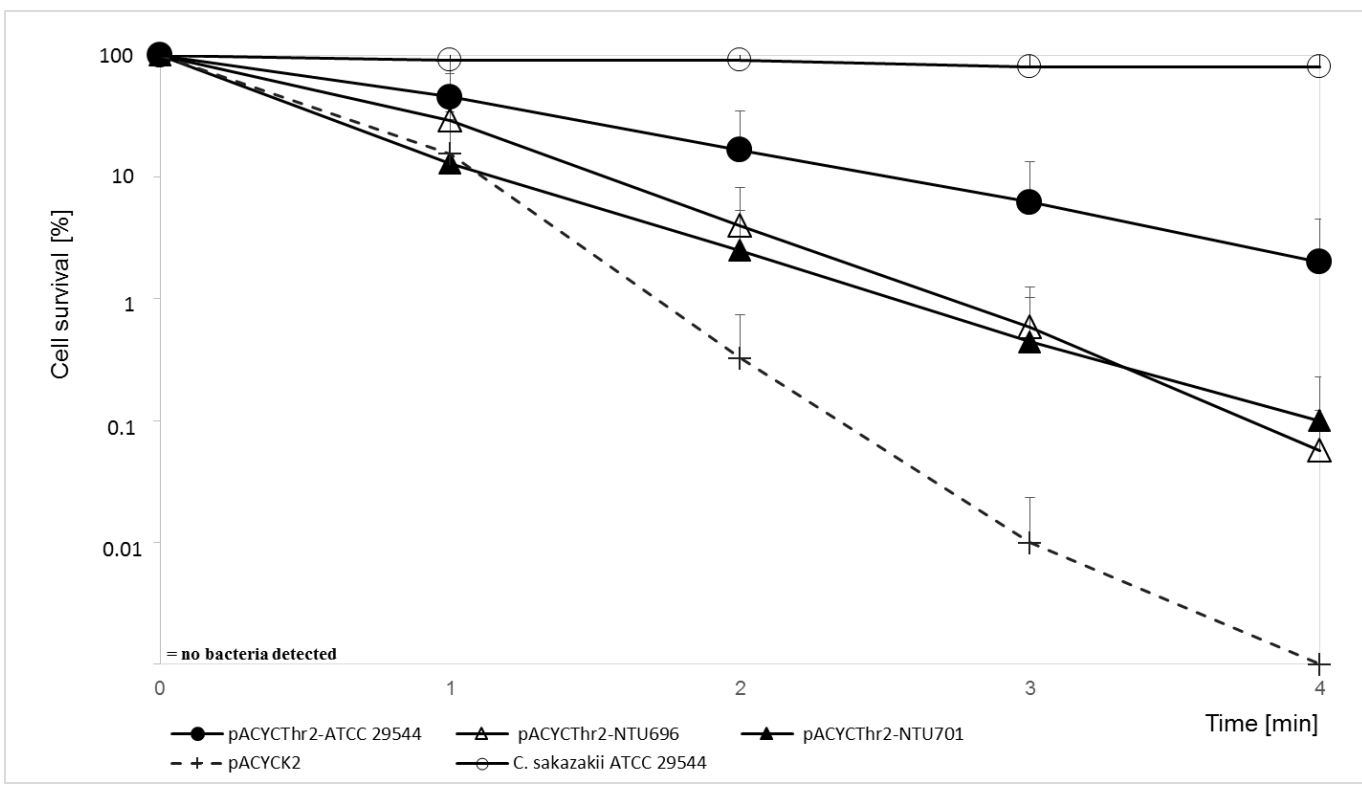

B) E. coli DH5 $\alpha$ was transformed with plasmids pACYC-ATCC29544 (filled circles), pACYCNTU701 (filled triangles) and pACYC-NTU696 (empty triangles) encoding for the thermotolerance islands from the respective Cronobacter strains (Fig. 1). Empty vector pACYCK2 (crosses) was used as negative control. Survival of stationary phase cells was determined at $58^{\circ} \mathrm{C}$.

$\mathrm{B}$

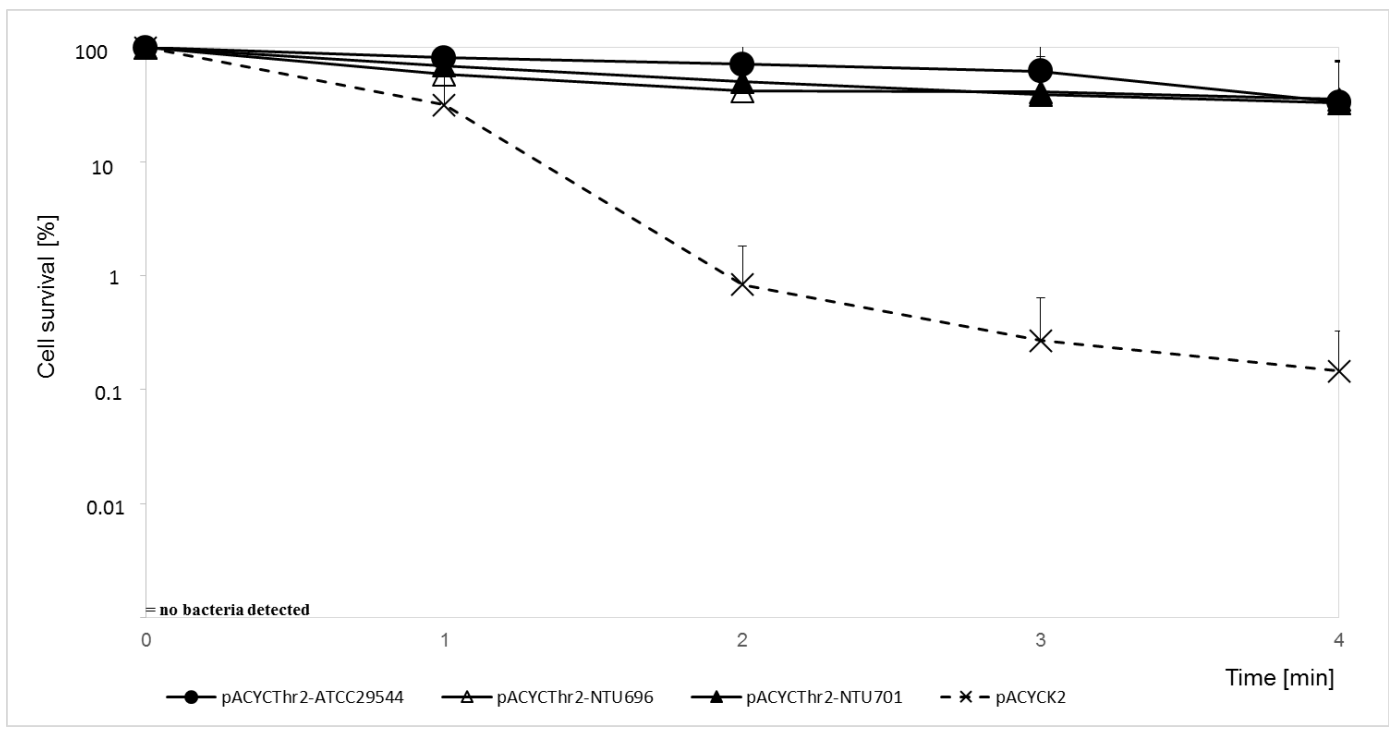


Fig 4. Influence of rpoS expression on thermotolerance

C. sakazakii NTU696 was transformed with plasmid pBAD-rpoS (filled triangles) encoding for the rpoS gene from E. coli KL53 under arabinose promoter. C. sakazakii NTU701 (filled circles) and C. sakazakii NTU696 (empty triangles) were used as positive and negative controls, respectively. Survival of stationary phase cells was determined at $58^{\circ} \mathrm{C}$.

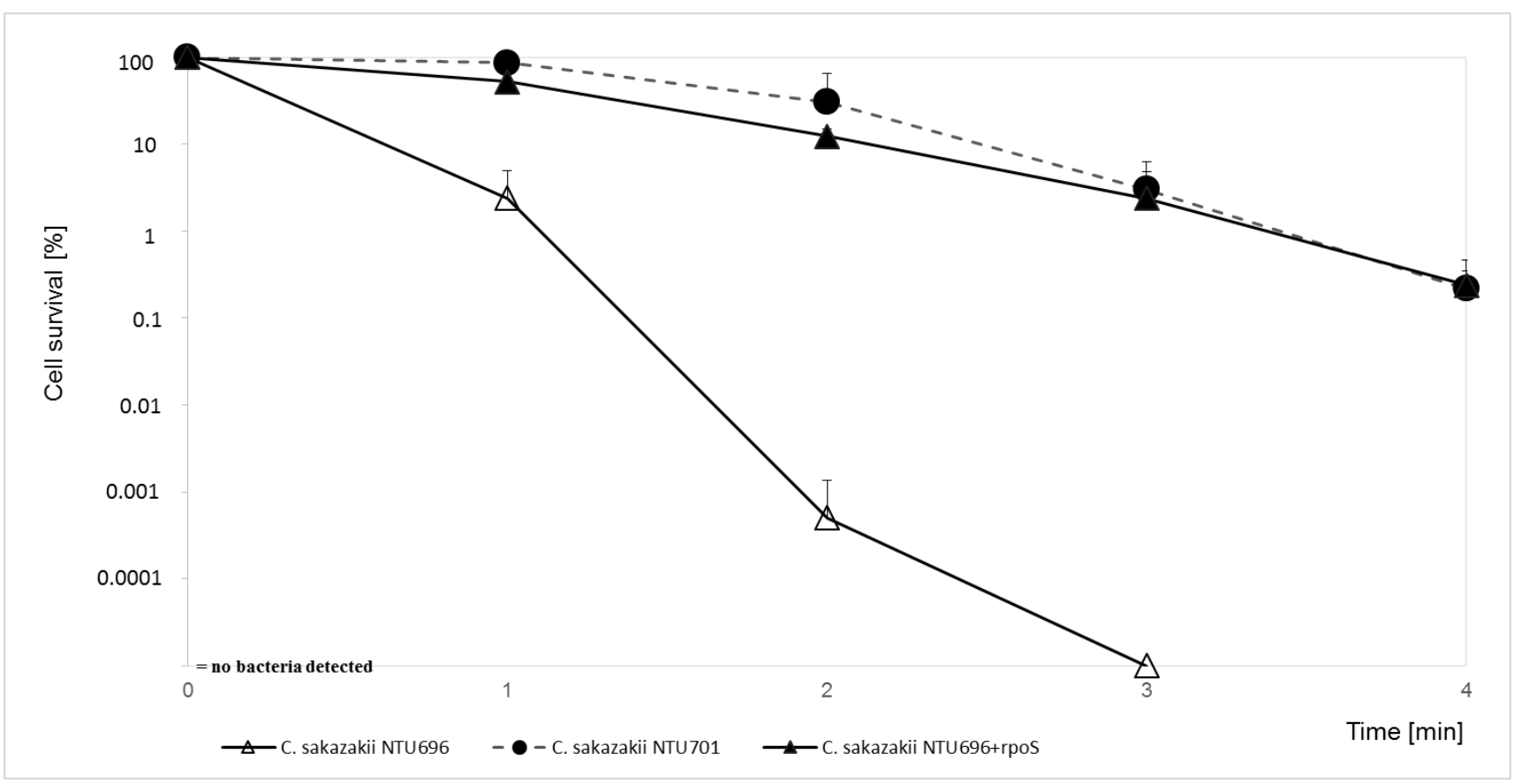


Fig 5. Thermotolerance of Cronobacter clinical isolates

Labelling of the strains: clinical C. sakazakii strains with the thermotolerance island (solid black lines), clinical C. malonaticus strains with the thermotolerance island (solid grey lines), the only clinical C. sakazakii strain lacking the thermotolerance island (217, solid black line with circles). C. sakazakii ATCC $29544^{\mathrm{T}}$ (dot chain line) and C. sakazakii JG-05 (dot line) were used as positive and negative controls, respectively. Survival of stationary phase cells was determined at $58^{\circ} \mathrm{C}$.

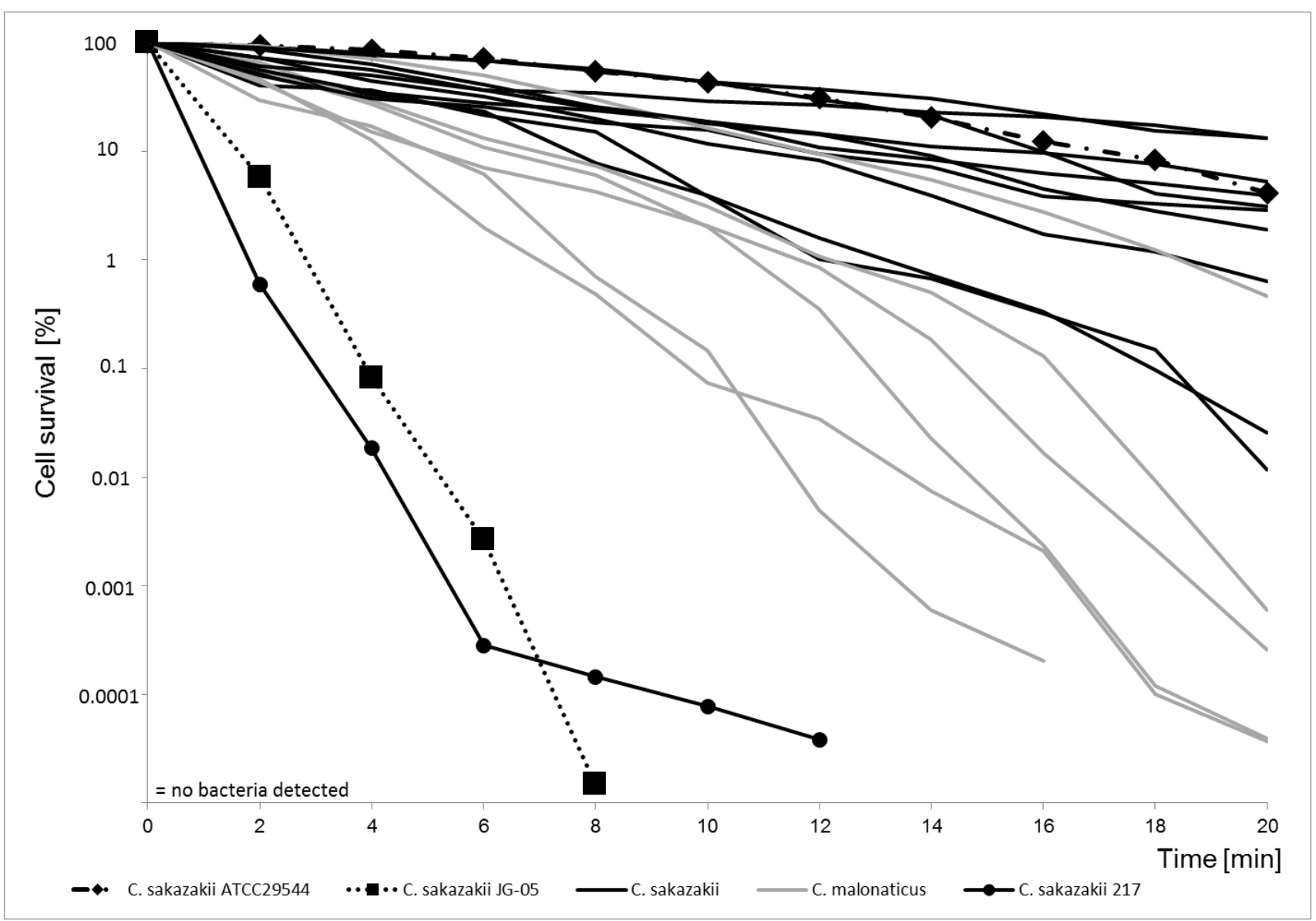

